\title{
A Time Decay Vacuum Is a New Era of Space Science with Reference to Magnetohydrodynamic Flows
}

\author{
Swapan Kumar Ghosh \\ Department of Mathematics, Narajole Raj College, Narajole, India \\ E-mail: g_swapan2002@yahoo.com \\ Received March 27, 2011; revised April 26, 2011; accepted May 6, 2011
}

\begin{abstract}
A controlled thermonuclear fusion reaction of the Sun is the heart of the space craft propulsion system. A band of ultraviolet radiation and X-ray emission of shorter wave length permeated by a magnetic mirror in the presence of a radiofrequency accelerator with the Sun at the resonant level is to produce hydrogen fusion laser due to a driving force to act as a laser fusion thruster. A superfluid helium $\left({ }^{4} \mathrm{He}_{2}\right)$ of burning with the Sun is a fifth state of matter. A higher state of matter is confined to a typical helical line which corresponds to the existence of outer universe.
\end{abstract}

Keywords: Radiofrequency Accelerator, Magnetic Mirror, Charged Oscillator, Microwave Background of Radiation, Fifth State of Matter

\section{Introduction}

Sun is a thermonuclear reactor. A radiofrequency accelerator with the Sun permeated by a magnetic mirror exhibits thermonuclear fusion reaction of the Sun propelled by $\mathrm{E} \gg \mathrm{mc}^{2}$ where $\mathrm{E}$ is the energy, $\mathrm{m}$ is the mass and $\mathrm{c}$ is the velocity of light. Since a radiofrequency accelerator is applied to the Sun, the frictional layer breaks down and the Sun is an exposure of a vacuum to produce radio wave. Radio emission is propagated from the Sun as the emission of ultra-relativistic electrons gyrating in a magnetic field. Finally, it comes to a conclusion that radio emission is a synchrotron radiation. A grand unified theory deals with the symmetry of the system in such a way that a controlled thermonuclear fusion reaction of the Sun communicates a band of ultraviolet radiation and $\mathrm{X}$-ray emission of shorter wave length at the resonant level in the presence of an oscilloscope to produce laser rainbow. This comes to a justification of a driving force at the resonant level where magnetic force $\left(\mathrm{M}^{2}\right)$ and Coriolis force $\left(\mathrm{K}^{2}\right)$ are of comparable in magnitude subject to the resonant condition $\omega>1 / 2 \cos \omega T\left(16 \mathrm{~K}^{4}-\right.$ $\left.\mathrm{M}^{4} \sin ^{4} \theta\right)^{1 / 2}$ with reference to a turbulent dynamo action of the universe. This condition is satisfied under grand unified theory when the angular frequency of oscillation $\omega T=\pi / 2$ at resonance to exerts its influence of a driving frequency $\omega>0$. A remarkable investigation on controlled thermonuclear fusion reaction of the Sun at the resonant level has been carried out by Ghosh [1], Ghosh and Pop [2,3], Ghosh [4-6], Ghosh et al. [7] and Ghosh and Beg [8]. However, a radiofrequency accelerator with the Sun permeated by a magnetic mirror can be exposed in such a way that laser radiation is so intense at the transition to produce multiphoton so that many photons can be simultaneously involved in a transition. A bright blue flash and a loud bang are produced and ultraviolet radiation and binary $\mathrm{X}$-rays are emitted as the air breaks down (In a Vacuum) to form an ionized plasma. A radiofrequency resonance at shorter wavelength exhibits bright blue flash (X-ray emission) and if we increase radiofrequency voltage (Increase of driving force that exceeds critical value) then $\mathrm{X}$-emission can be transformed into $\mathrm{X}$-ray burst with thermonuclear flashes. Although a thermonuclear fusion reaction of the Sun deals with $\mathrm{X}$-ray photon in a vacuum. A thermonuclear fusion reaction in a radiofrequency field exhibits two types of X-ray regime viz., soft X-rays and hard X-rays. Soft X-rays can be defined by short $\mathrm{X}$-ray burst due to thermonuclear flashes at long wave length and the hard X-ray burst comes from the synchronization of ultraviolet radiation and X-ray emission at shorter wave length with a significant effect of air friction (radio wave). A radio wave in a vacuum deals with solar wind to become a solar flare as generated from the Sun.

A controlled thermonuclear fusion reaction of the Sun at the resonant level is the heart of a space craft propul- 
sion system. Plasma fusion interacts with the supercritical state in the presence of a radiofrequency accelerator subject to an oscillating magnetic field propelled by magnetic pumping which becomes important to an application of plasma fusion thruster with significant effect of pressure gradient. In turn, a convective part of the surface of the Sun becomes important to produce hydrogen fusion laser under thermonuclear confinement in the presence of a magnetic mirror with the Sun permeated by a charged oscillator. This situation reveals that the reflection occurs with an abrupt increase in magnetic field strength in the presence of a charged oscillator so that magnetic field changes its direction from the central region due to buoyancy of the convective part of the surface of the Sun and eventually, this investigation turns into a high energy laser fusion thruster to become a new generation of a space craft propulsion system subject to the resonant condition $\omega>1 / 2 \cos \omega T\left(16 \mathrm{~K}^{4}-\mathrm{M}^{4} \sin ^{4} \theta\right)^{1 / 2}$ with reference to a driving frequency $\omega>0$.

A radiofrequency accelerator with the Sun in the presence of a magnetic mirror is an exposure of a controlled thermonuclear fusion reaction at the resonant level to produce radio isotope in the electromagnetic field to form electron cloud particle and thermonuclear fusion takes place of rain fall with an irregular fluctuation of electrons in a microgravity field. This indicates that radio wave determines artificial rain fall in a microgravity field to become relevant to a crystal growth in space. A controlled thermonuclear fusion reaction of the Sun leads to a plasma discharge of a microgravity field with different colors in the presence of crystal laser. A crystal laser of different colors refracts with different wave length. In the light of crystal laser, it is worth mentioning that crystalline substance interacts with different color of laser light and ionization takes place with sparking of laser rays of different wave length in a microgravity field.

Nevertheless, nuclear bombardment with ionized hydrogen is subjected to a hydrogen bomb blasting on the surface of the Sun so that maximum dissipation of energy is liberated from surface of the Sun at the smallest eddies when the kinetic energy is transformed into heat and an abundance of helium is produced. In this regard, an abundance of helium with the Sun is the transformation of superfluid helium $\left({ }^{4} \mathrm{He}_{2}\right)$ of burning to condensate at a very low temperature. It experimentally verified that if we freeze the condensate matter then the temperature $0^{0}$ falls to $-2^{0} \mathrm{~K}$ to become a superfluid. This situation arises in such a way that the condensation of matter becomes no frictional force which is a good agreement with time decay vacuum. Therefore, the configuration of the Sun is a fifth state of matter due to a superfluid helium $\left({ }^{4} \mathrm{He}_{2}\right)$ of burning to become a low temperature. Owing to condensate the superfluid helium $\left({ }^{4} \mathrm{He}_{2}\right)$ of burning, crystal growth becomes significant to the case of a microgravity field. In fact, a condensate matter can be recognized from the superfluid helium $\left({ }^{4} \mathrm{He}_{2}\right)$ of burning in such a way that if the temperature of the surrounding fluid is zero then its surface temperature should be zero. Also the rate of heat transfer becomes insignificant. Since grand unified theory determines a big bang to the symmetry of the system, the breaking of symmetry with reference to hot big bang subject to $\mathrm{E} \gg \mathrm{mc}^{2}$ corresponds to the three independent pieces of evidences viz., 1) the expansion of universe, 2) microwave background of radiation and 3 ) the abundance of helium.

\section{In Such a Case an Interesting Mathematical Relation for Heat Transfer Is Developed}

Consider the steady MHD free convection boundary layer flow of a viscous, incompressible, electricallyconducting Newtonian fluid past an infinite, vertical, flat plate, directed along the positive $x^{\prime}$-axis, in the presence of an applied uniform transverse magnetic field. It is assumed that the plate is isothermal where the constant surface temperature $T_{w}$ of the plate is exposed to the fluid. The temperature of the surrounding fluid is $T_{o}$ at a distance from the plate. A layer of the ascending heated fluid appears at the plate. The lower edge is taken as the origin of the leading edge of the plate and the $y^{\prime}$-axis is orientated in the direction perpendicular to its surface. A uniform magnetic flux, $B_{0}$, is applied parallel to the $y^{\prime}$-axis. Since the plate is infinite along the $x$ '-direction, all physical quantities will be function of the independent variable, $y^{\prime}$, only. The following assumptions are inherent in our study:

1) The force of inertia is negligible as compared with those of gravity and viscosity.

2) The transport of heat by conduction and convection along the moving layer of the fluid can be ignored.

3) The pressure gradient is zero.

4) The physical parameters of the fluid (except density) are constant and density is a linear function of temperature.

We assume that in the moving layer, temperature varies under the following condition:

$$
\frac{T-T_{o}}{T_{w}-T_{o}}=\left(1-\frac{y}{\delta}\right)^{2}
$$

where $\psi=T-T_{o}$ and $\psi_{w}=T_{w}-T_{o}$. In the present investigation, the temperature profile can be described in the moving layer with reference to $T_{w}-T_{o}=$ constant. Under assumption (1) the following boundary conditions are satisfied:

$$
\psi=\psi_{w} \text { at } y=0
$$




$$
\psi=0 \text { at } y=\delta
$$

Here, $T$ is the temperature in the reference state, $T_{o}$ is the temperature of the surrounding fluid and $T_{w}$ is the surface temperature.

In special case, if the temperature of the surrounding fluid $T_{o}$ is zero then the condensation of matter with reference state of temperature $T$ is zero and therefore, the surface temperature $T_{w}$ of the medium is zero. Then, specific heat at constant temperature is zero for the case of Prandtl number $P_{r}=\frac{v}{\alpha_{1}}$ where $\alpha_{1}=\frac{K}{\rho C_{p}}$. Here, $\mathrm{K}$ is the thermal conductivity, $\rho$ is the fluid density and $v$ is the kinematic coefficient of viscosity.

In relevance to the physical situation of interest it is more convincing to say that a time decay vacuum is a decisive importance to an electromagnetic field when mass of an electron is negligible in a force free field.

\section{Determination of the Validity of an Argument under Electromagnetic Field}

The Maxwell's equations are

$$
\begin{gathered}
\nabla \times B=\mu_{e} J \quad \text { (Ampère's Law) } \\
\nabla \times E=-\frac{\partial B}{\partial t} \quad \text { (Faraday's Law) }
\end{gathered}
$$

$\nabla \cdot B=0 \quad$ (Solenoidal relation)

$\nabla \cdot J=0 \quad$ (Gauss's Law i.e. Conservation of electric charge)

In special case when the displacement current is present, $\nabla \cdot J \neq 0$ corresponds to a time varying magnetic field of sinusoidal in nature to become a fluctuation at every point on the flow field (see Ghosh et al. [7]) and this situation becomes relevant to the case of traveling magnetic field.

$B, E, J, \mu_{e}$ and $t$ are, respectively, the magnetic field vector, electric field vector, current density vector, magnetic permeability and time.

The differential equation which governs the motion of charged particles results from introducing the electric and magnetic forces into Newton's Second law. The equation of motion for a particle of mass $\mathrm{m}$ and charge $\mathrm{q}$

$$
\frac{\mathrm{d} V}{\mathrm{~d} t}=\frac{q}{m}[E+V \times B]
$$

The Ohm's law for a moving conductor becomes

$$
J=\sigma[E+V \times B]
$$

where $V$ is the velocity vector and $\sigma$ is the electrical conductivity

Comparing (4) and (5) it turns into

$$
J=\frac{m \sigma}{q} \frac{\mathrm{d} V}{\mathrm{~d} t}
$$

On using (6) if the mass of an electron $m$ is zero then the current density

$J=0$. Therefore, $\sigma$ tends to zero.

$$
\text { It reveals that } \nabla \times H=0
$$

where $H$ is the intensity of a magnetic field vector.

The equation (7) can be transformed into

$$
\nabla \times B=0
$$

where $B=\mu_{e} H$.

Since the force free field is associated with $\nabla \times B=0$ there exists a scalar function of position $K(x)$ such that

$$
\nabla \times B=K B, \quad B \nabla K=0
$$

In this situation $\nabla \cdot B=0$ can be found from second equation of (9) where $K$ is therefore constant on $B$-lines and if $B$-lines cover surfaces then $K$ must be constant on each surface. In this case taking curl of (9) immediately leads to the Helmholtz equation

$$
\left[\nabla^{2}+K^{2}\right] B=0
$$

Equation (10) indicates that in a force free field there exists a potential flow to determine zero frictional force. This corresponds to a time decay vacuum of a current free zone surrounding the medium. An interesting situation appears to be a significant manner in such a way that the time decay vacuum is the representation of an invisible object whose mass is negligibly small. In such a situation if we recognize an object then it is true, On the other hand, if we do not see the object then we can not find the behavior and properties of an unrecognized object. In a practical situation of interest it is stated that a time decay vacuum is a secret communication of an invisible matter to deal with outer space. In the light of a fifth state of matter it is rigorously stated that a supernatural power comes from the typical helical line and the existence of outer universe becomes significant.

In turn, it appears to be a current free zone surrounding the condensate matter in a vacuum when mass is negligibly small. In this context, a typical helical line becomes relevant to the case of a cosmological background of outer space. An interesting situation is observed from the above discussions that the soul can not be destroyed and a typical helical line communicates with outer space under the influence of a radiofrequency to activate the kinetic energy of the system. In this case the fifth state of matter can be transformed into a higher state of matter.

\section{Conclusions}

A controlled thermonuclear fusion reaction of the Sun becomes relevant to a study of magnetohydrodynamics (MHD) with reference to space craft propulsion system. In the light of a study of magnetohydrodynamics it is 
rigorously stated that the superfluid helium $\left({ }^{4} \mathrm{He}_{2}\right)$ of burning is a fifth state of matter to condensate at low temperature of negligible mass. Eventually, if mass is negligibly small there arise a force free field of a current free zone surrounding the medium but it appears to be a typical helical line of a time decay vacuum.

\section{References}

[1] S. K. Ghosh, "A Note on Unsteady Hydromagnetic Flow in a Rotating Channel Permeated by an Inclined Magnetic Field in the Presence of an Oscillator," Czechoslovak Journal of Physics, Vol. 51, No. 8, 2001, pp. 799-804. doi:10.1023/A:1011622416362

[2] S. K. Ghosh and I. Pop, "Comments on MHD Plasma Flow in a Rotating Environment in the Presence of an Inclined Magnetic Field," International Journal of Applied Mechanics and Engineering, Vol. 9, No. 4, 2004, pp. 821 $-826$.

[3] S. K. Ghosh and I. Pop, "An Analytical Approach to MHD Plasma Behavior of a Rotating Environment in the Presence of an Inclined Magnetic Field as Compared to Excitation Frequency," International Journal of Applied Mechanics and Engineering, Vol. 11, No. 4, 2006, pp. 845-856.
[4] S. K. Ghosh, "Report on Plasma Behavior over the Interstellar Medium," International Journal of Applied Mechanics and Engineering, Vol. 11, No. 4, 2006, pp. 981983.

[5] S. K. Ghosh, "Exploitation of the Universe with Regard to Controlled Thermonuclear Fusion Reaction of the Sun," International Journal of Applied Mechanics and Engineering, Vol. 12, No. 2, 2007, pp. 571- 573.

[6] S. K. Ghosh, "Transmission of Radio Wave with Regard to Controlled Thermonuclear Fusion Reaction of the Sun," Advanced Studies in Theoretical Physics, Vol. 1, No. 4, 2007, pp. 169-172.

[7] S. K. Ghosh, O. A. Beg, J. Zueco and V. R. Prasad, "Transient Hydromagnetic Flow in a Rotating Channel Permeated by an Inclined Magnetic Field with Magnetic Induction and Maxwell Displacement Current Effects," Zeitschrift Angewandte Mathematik und Physik: ZAMP, Vol. 61, No. 1, 2010, pp. 147-169. doi:10.1007/s00033-009-0006-2

[8] S. K. Ghosh and O. A. Beg, "A Note on Thermonuclear Hot Big Bang with Reference to Controlled Fusion of the Sun," Journal of Magnetohydrodynamics, Plasma and Space Research, Vol. 15, No. 2, 2010, pp. 1-5. 